\title{
Risk of cancer in patients with thyroid disease and venous thromboembolism
}

This article was published in the following Dove Press journal:

Clinical Epidemiology

\author{
Diana H Christensen' \\ Katalin Veres' \\ Anne G Ording' \\ Jens Otto L Jørgensen ${ }^{2}$ \\ Suzanne C Cannegieter ${ }^{3}$ \\ Reimar W Thomsen' \\ Henrik T Sørensen' \\ 'Department of Clinical Epidemiology, \\ Aarhus University Hospital, \\ Aarhus, Denmark; ${ }^{2}$ Department of \\ Endocrinology and Internal Medicine, \\ Aarhus University Hospital, Aarhus, \\ Denmark; ${ }^{3}$ Department of Clinical \\ Epidemiology, Leiden University \\ Medical Centre, Leiden, the \\ Netherlands
}

Correspondence: Diana H Christensen Department of Clinical Epidemiology, Aarhus University Hospital, Olof Palmes Allé 43-45, DK-8200, Aarhus N, Denmark

Tel +4587168248

Fax +4587167215

Email dhcr@clin.au.dk
Objective: Risk of venous thromboembolism (VTE) is increased in patients with hypo/ hyperthyroidism. It is unknown whether VTE may be a presenting symptom of occult cancer in these patients.

Design: Nationwide population-based cohort study based on Danish medical registry data. Methods: We identified all patients diagnosed with VTE during 1978-2013 who had a previous or concurrent diagnosis of hypothyroidism $(\mathrm{N}=1481)$ or hyperthyroidism $(\mathrm{N}=1788)$. We followed them until a first-time cancer diagnosis, death, emigration, or study end, whichever came first. We calculated 1-year absolute cancer risk and standardized incidence ratios (SIRs) for cancer incidence in the study population compared with national cancer incidence in the general population.

Results: During the first year after a VTE diagnosis, the 1-year absolute cancer risk was 3.0\% among patients with hypothyroidism and 3.9\% among those with hyperthyroidism. During the first year of follow-up, SIRs for cancer in the study population compared with the general population were 1.96 (95\% CI: 1.42-2.64) among patients with hypothyroidism and 2.67 (95\% CI: 2.07-3.39) among those with hyperthyroidism. SIRs declined substantially after 1 year but remained increased during the remainder of the follow-up period (up to 36 years) (SIR for hypothyroidism=1.16 [95\% CI: 0.97-1.39]; SIR for hyperthyroidism=1.26 [95\% CI: $1.08-1.46]$ ). Conclusion: VTE may be a marker of underlying occult cancer in patients with hypothyroidism or hyperthyroidism.

Keywords: hyperthyroidism, hypothyroidism, venous thromboembolism, cancer, cohort study

\section{Introduction}

Hyperthyroidism and hypothyroidism are common endocrine diseases, with estimated lifetime risks of $2 \%-5 \% .{ }^{1,2}$ Hyperthyroidism is associated with biochemical changes consistent with vascular endothelial dysfunction and hypercoagulability -2 of the 3 factors that comprise Virchow's triad of pathophysiological factors in thrombosis development. ${ }^{3,4}$ Moreover, hyperthyroidism is associated with reduced fibrinolytic activity. ${ }^{3,4}$ Accordingly, several cohort and case-control studies have reported up to a 6-fold increased risk of venous thromboembolism (VTE) in patients with hyperthyroidism, ${ }^{5-10}$ with the increased VTE risk persisting for several years after diagnosis. ${ }^{5}$

The relation between hypothyroidism and coagulation disturbances is less clear. Some studies have noted bleeding tendencies and others have reported a hypercoagulable and hypofibrinolytic state. ${ }^{11}$ VTE risk in patients with hypothyroidism has been poorly investigated, with a single observational study reporting a 1.6-fold increased risk. ${ }^{12}$ 
VTE is a well-known and frequent cancer complication. ${ }^{13}$ Moreover, VTE occurrence may be the first sign of an as yet undiagnosed cancer. ${ }^{14-19}$ Previous studies have found a 2- to 4-fold increased 1-year risk of cancer among patients diagnosed with VTE compared with the general population. ${ }^{14-18}$ Initially, this was thought to be relevant only for primary or idiopathic VTE, ${ }^{19}$ that is, VTE occurring with no preceding risk factors, but increasing evidence suggests that occult cancer also may be a contributing cause in patients with secondary VTE. ${ }^{14}$

It is unknown whether VTE in patients with thyroid disease may be a marker of undiagnosed cancer. We, therefore, conducted this nationwide Danish population-based cohort study to compare cancer risk following VTE among patients with thyroid disease with that expected based on national cancer incidence.

\section{Materials and methods}

\section{Setting and data sources}

The Danish social welfare system provides tax-funded health care to the entire Danish population, with all provided services registered in national health care databases. Accurate linkage of these databases is possible via the unique civil registration number (CPR number) assigned to each Danish resident at birth or upon emigration. ${ }^{20}$ The source population of the current cohort study consisted of the entire Danish population. During the 36-year study period (January 1, 1978 to November 30,2013 ), the cumulative population included $8,096,820$ persons.

\section{Cohort of patients with VTE and thyroid disease}

The Danish National Patient Registry (DNPR) contains information on all non-psychiatric inpatient admissions in Denmark since 1977. Hospital outpatient clinic visits and emergency room visits were added in $1995 .{ }^{21}$ Information recorded in the DNPR includes the CPR number, dates of admission and discharge, outpatient/emergency room visit dates, surgical procedures performed, and discharge diagnoses classified according to the International Classification of Diseases, Eighth Revision through 1993 and Tenth Revision (ICD-10) thereafter. ${ }^{21}$ We used the DNPR to identify all patients with a first-time VTE (deep venous thrombosis of the lower limb or pulmonary embolism) diagnosed during an inpatient admission or hospital outpatient clinic visit during the study period $(\mathrm{N}=156,387)$. Both primary and secondary discharge diagnoses were included. In Denmark, diagnoses of first-time VTE have a positive predictive value (PPV) higher than $80 \% .{ }^{22}$ We excluded patients with VTEs diagnosed in the emergency room setting without a subsequent inpatient diagnosis, because of the low PPV (31\%) of these diagnoses. ${ }^{23}$ We also excluded all VTE patients who had a cancer diagnosis recorded at any time before or during the hospital contact with VTE $(\mathrm{N}=32,778)$. We then restricted our study cohort to VTE patients with a diagnosis of hypothyroidism $(\mathrm{N}=1481)$ or hyperthyroidism $(\mathrm{N}=1788)$ recorded at any time before or during the hospital contact in which the VTE was diagnosed. Patients with ICD-10 codes for myxedema after treatment or thyrotoxicosis by overdose of thyroid hormone were excluded, because these patients could not clearly be assigned to either the hypo- or hyperthyroidism cohort. In the subgroup of VTE patients $(\mathrm{N}=226)$ who had both hypothyroidism and hyperthyroidism diagnoses recorded, the most recent pre-VTE thyroid disease diagnosis code determined membership in the hypothyroidism vs hyperthyroidism subcohort. This was done to ensure that subcohort membership reflected thyroid hormone levels closest in time to the VTE date.

\section{Cancer outcomes}

We obtained information on cancers from the Danish Cancer Registry, which has recorded all incident cancers in Denmark since 1943, with information on morphology, histology, and cancer stage at diagnosis. ${ }^{24}$ We used the same grouping of cancers as presented in the Annual Cancer Report published by the Danish National Board of Health.

\section{Covariates}

The inpatient and outpatient hospital history available in the DNPR provided information on classic VTE risk factors in the 90-day period prior to VTE diagnosis (surgery, fractures/ trauma, and pregnancy), on obesity, and on diseases included in the Charlson Comorbidity Index (CCI). ${ }^{25,26}$ The CCI assigns $1-6$ points to 19 disease categories according to their ability to predict short-term mortality. Based on total modified CCI scores (cancer excluded), we defined 3 categories of comorbidity burden: normal (0 points), moderate (1-2 points), and high (3 or more points).

We obtained information on vital status from the Civil Registration System, which records data on death and migration with daily electronic updates. ${ }^{20}$

Relevant ICD codes and supporting information about grouping of cancers are provided in the online supplementary data (Table S1). 


\section{Statistical analyses}

We followed patients from VTE diagnosis until a cancer diagnosis, death, emigration, or study end, whichever occurred first. The follow-up period was split into $0-1$ year and $1+$ years. The first year was further divided into 0-90 days and 91-365 days.

We calculated 1-year absolute cancer risks for all cancers, treating death as a competing risk. ${ }^{27}$ We calculated the expected cancer rate among patients with hypo/hyperthyroidism and VTE, assuming the expected cancer risk in this population would be the same as in the general population. We multiplied the number of person-years of observation by the Danish national cancer incidence rates across gender, single-year age groups, and single-year periods of diagnosis year to achieve the expected number of incident cancers. We then calculated the standardized incidence ratio (SIR), that is, the ratio of the observed to the expected number of cancers. This served as a measure of the relative risk of cancer in patients with thyroid disease and VTE. We calculated 95\% CIs under the assumption that the observed number of cancers in a specific category followed a Poisson distribution. ${ }^{28}$ Exact 95\% CIs were used when the observed number of cancers was $<10$; otherwise, Byar's approximation was used. ${ }^{28}$

Assuming that cancers detected during the first year following VTE also were present at the time of VTE diagnosis, we calculated the reciprocal of the excess risk ([observed number of cancers/follow-up time] - [expected number of cancers/follow-up time]) for the first year of follow-up, in order to determine the number needed to examine at time of VTE to detect 1 excess cancer. ${ }^{29}$ As well, 95\% CIs were calculated as the reciprocal of the CIs for the excess risk estimate. ${ }^{30}$

In subgroup analyses, the results were stratified according to age at VTE diagnosis, calendar-year period of VTE diagnosis (1978-1993 and 1994-2013), gender, comorbidity burden, obesity, and presence/absence of classic VTE risk factors.

In this study, we excluded all VTEs preceded by a cancer diagnosis. However, there may have been some delay in recording the cancer diagnosis. In addition, VTEs could have been detected coincidentally during diagnostic workup in patients suspected to have cancer. In order to examine the potential impact of including such VTEs in the analysis, the 90-day follow-up period was divided into 0-30 days and 31-90 days in sensitivity analyses. We also repeated all analyses after excluding cancers detected within the first 30 days. Moreover, we repeated all analyses excluding the 226 patients recorded as having both hypothyroidism and hyperthyroidism. Pharmacological or - for hyperthyroidism
- surgical treatment may change hormone status to euthyroid. Therefore, we also conducted a sensitivity analysis in which we restricted the cohort to patients with a maximum 2-year interval between their first hypo/hyperthyroid disease diagnosis and their first VTE diagnosis in order to increase the likelihood that our study population reflects patients with ongoing thyroid disease.

Statistical analyses were conducted using the SAS statistical software package, version 9.4 (SAS Institute, Cary, NC, USA). This study did not involve any patient contact or any intervention. Thus, approval from the Danish Scientific Ethical Committee and patient consent were not required. The study was approved by the Danish Data Protection Agency (record number 1-16-02-1-08).

\section{Results Descriptive data}

We identified 1481 patients with hypothyroidism (86\% female, median age: 75 years) and 1788 patients with hyperthyroidism ( $83 \%$ female, median age: 74 years) with a first-time VTE diagnosis (Table 1). Median time from first thyroid disease diagnosis to VTE diagnosis was 4.1 years (interquartile range $[\mathrm{IQR}]=0.6-10.0$ years). The hypothyroidism subcohort was followed for a median of 2.5 years (IQR $=0.4-6.1$ years) and the hyperthyroidism subcohort was followed for a median of 2.6 years (IQR $=0.3-6.6$ years). In both subcohorts, $24 \%-25 \%$ of patients had classic risk factors and $\geq 78 \%$ of patients were diagnosed with VTE in the second half of the study period (1994-2013). Hospital-coded obesity was relatively uncommon $(6 \%-11 \%)$ and was most prevalent among patients with hypothyroidism. Hospital-diagnosed comorbidity, defined as CCI points $\geq 1$, was observed in $65 \%$ of patients with hypothyroidism and in $56 \%$ of those with hyperthyroidism.

\section{Cancer data}

During follow-up, 164 cancers were diagnosed among patients with hypothyroidism and 239 among those with hyperthyroidism. In the hypothyroidism subcohort, the 1 -year absolute cancer risk was $3.0 \%$, corresponding to a 1-year cancer SIR of 1.96 (95\% CI: 1.42-2.64). In the hyperthyroidism subcohort, the 1-year absolute cancer risk was $3.9 \%$, corresponding to a 1-year cancer SIR of 2.67 (95\% CI: 2.07-3.39). Cancers were diagnosed most often within the first 90 days after a VTE diagnosis. In the hypothyroidism subcohort, the 90-day cancer SIR was 2.36 (95\% CI: $1.29-3.96)$, the 91-365 days SIR was 1.81 (95\% CI: 1.21-2.60), and the SIR during 1+ years of follow-up was 
Table I Characteristics of patients with thyroid disease and a VTE, Denmark, 1978-2013

\begin{tabular}{|c|c|c|}
\hline & Hypothyroidism & Hyperthyroidism \\
\hline & $\mathbf{N}(\%)$ & $\mathbf{N}(\%)$ \\
\hline Total & $148 \mid(100)$ & $1788(100)$ \\
\hline Female & $1278(86)$ & $1480(83)$ \\
\hline Median age at VTE diagnosis (IQR), years & $75(65-82)$ & $74(64-82)$ \\
\hline \multicolumn{3}{|l|}{ Age at VTE diagnosis } \\
\hline$<60$ years & $259(17)$ & $326(18)$ \\
\hline $60-74$ years & $488(33)$ & $598(33)$ \\
\hline $75+$ years & $734(50)$ & $864(48)$ \\
\hline \multicolumn{3}{|l|}{ Comorbidity burden ${ }^{\mathrm{a}}$} \\
\hline Normal & $516(35)$ & 789 (44) \\
\hline Medium & $673(45)$ & $762(43)$ \\
\hline High & $292(20)$ & $237(13)$ \\
\hline \multicolumn{3}{|l|}{ Charlson Comorbidity Index conditions } \\
\hline Myocardial infarction & $167(\mathrm{II})$ & $158(9)$ \\
\hline Congestive heart failure & $247(17)$ & $281(16)$ \\
\hline Peripheral vascular disease & $145(10)$ & $172(10)$ \\
\hline Cerebrovascular disease & $251(17)$ & $275(15)$ \\
\hline Dementia & $56(4)$ & $46(3)$ \\
\hline Chronic pulmonary disease & $302(20)$ & $312(17)$ \\
\hline Connective tissue disease & $192(13)$ & $124(7)$ \\
\hline Gastrointestional ulcer & $139(9)$ & $132(7)$ \\
\hline Mild liver disease & $39(3)$ & $19(1)$ \\
\hline Diabetes & $202(14)$ & $182(10)$ \\
\hline Hemiplegia & $8(1)$ & $7(0.4)$ \\
\hline Moderate-to-severe renal disease & $100(7)$ & $65(4)$ \\
\hline Diabetes with end-organ disease & $103(7)$ & $84(5)$ \\
\hline Moderate-to-severe liver disease & $7(1)$ & $3(0.2)$ \\
\hline AIDS & $0(0)$ & $0(0)$ \\
\hline \multicolumn{3}{|l|}{ Year of VTE diagnosis } \\
\hline $1978-1993$ & $318(21)$ & $397(22)$ \\
\hline |994-20II & $\mathrm{I}, \mathrm{I} 63(79)$ & I,39I (78) \\
\hline Obesity & $163(11)$ & $108(6)$ \\
\hline \multicolumn{3}{|l|}{ Provoking factors ${ }^{\mathrm{b}}$} \\
\hline Classic provoking factors, overall & $37 \mid(25)$ & $424(24)$ \\
\hline Surgery & $293(20)$ & $341(19)$ \\
\hline Trauma/fracture & $136(9)$ & $171(10)$ \\
\hline Pregnancy & $5(0.3)$ & $4(0.2)$ \\
\hline
\end{tabular}

Notes: ${ }^{a}$ Three categories of comorbidity burden based on the Charlson Comorbidity Index; normal=0 points, moderate $=1-2$ points, and high=3 or more points. Cancer was excluded from the comorbidity index. 'Within the 3 months prior to a VTE diagnosis.

Abbreviations: IQR, interquartile range; VTE, venous thromboembolism; AIDS, acquired immune deficiency syndrome.

1.16 (95\% CI: 0.97-1.39). A similar pattern was observed for the hyperthyroidism subcohort. However, the cancer risk within the first 90 days was even higher and the risk remained modestly increased beyond 1 year of follow-up. The 0-90 day cancer SIR was 5.32 (95\% CI: 3.75-7.33), the 91-365 day cancer SIR was 1.68 (95\% CI: 1.14-2.38), and the cancer SIR during 1+ years of follow-up was 1.26 (95\% CI: 1.08-1.46; Tables 2 and 3). The number of patients needed to examine in order to detect 1 excess cancer within the first year following VTE was 52 (95\% CI: 32-142) in the hypothyroidism subcohort and 30 (95\% CI: 22-49) in the hyperthyroidism subcohort.

\section{Subgroup analyses}

In the hypothyroidism subcohort, the 1-year cancer SIR was higher among patients who were younger and had a lower comorbidity burden. In contrast, the SIR showed only minor changes after stratification by gender, hospital-diagnosed obesity, calendar-year period, and presence/absence of classic VTE risk factors (Table 2).

In the hyperthyroidism subcohort, 1-year cancer SIRs varied modestly by age, comorbidity burden, gender, and calendar-year period (Table 3).

In the hypothyroidism subcohort, a 3-fold or greater increase was observed within the first year of follow-up for 


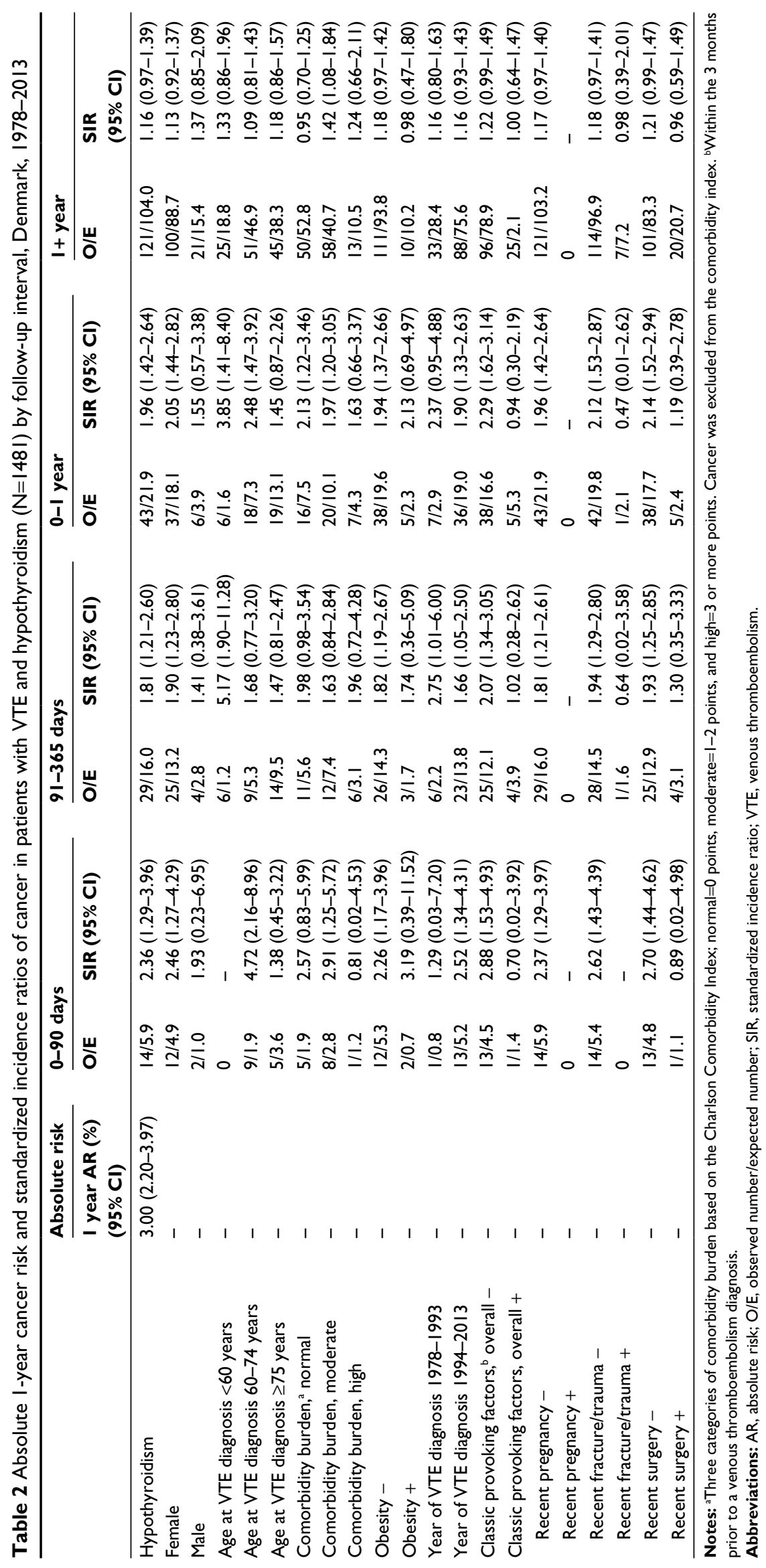




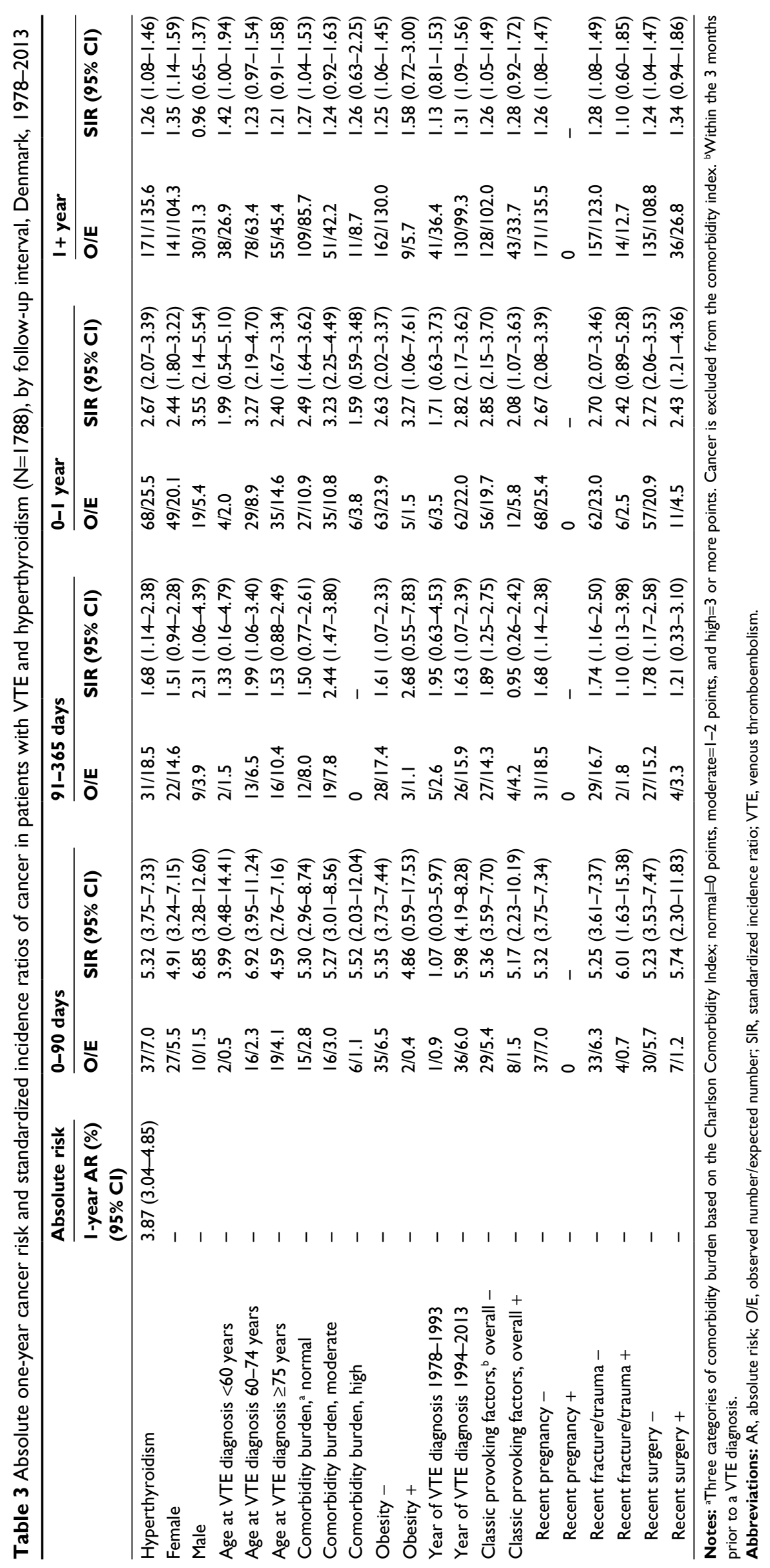


cancers of the ovary and for non-Hodgkin malignant lymphoma. In the hyperthyroidism subcohort, corresponding increases were observed for cancers of the large intestine, pancreas, uterine cervix, uterus, ovary, prostate, and urinary bladder, as well as for non-specified cancers and metastases (Tables S2 and S3).

\section{Sensitivity analyses}

Within 30 days following the VTE diagnosis date, 8 cancers were diagnosed in the hypothyroidism subcohort and 27 in the hyperthyroidism subcohort. The 30-day SIRs were 3.91 (95\% CI: 1.69-7.70) and 11.10 (95\% CI: 7.32-16.16), respectively, in the 2 subcohorts (Tables S4 and S5). Excluding these cancers, the 1-year SIR decreased to $1.76(95 \%$ CI: 1.23-2.45) in the hypothyroidism subcohort and to 1.78 (95\% CI: 1.28-2.42) in the hyperthyroidism subcohort. (Tables S6 and S7). Excluding the 226 patients with both hypo- and hyperthyroid diagnosis did not change the results (data not shown). Restricting the cohort to patients with a maximum interval of 2 years from hypo/hyperthyroid diagnosis to VTE diagnosis had no significant effect on the results for the hypothyroidism subcohort (Tables S8-S10). For the hyperthyroidism subcohort, the SIRs, in general, increased, except for the 91-365 days period; 0-90 days SIR: 8.27 (95\% CI: 4.90-13.08), 91-365 days SIR: 1.36 (95\% CI: 0.59-2.68), 1-year SIR: 3.22 (95\% CI: 2.11-4.72), and SIR during 1+ years of follow-up: 1.54 (95\% CI: 1.22-1.91; Tables S11-S13). However, precision was reduced in these sensitivity analyses.

\section{Discussion}

In this large population-based cohort study, we evaluated the association between VTE occurrence and subsequent cancer diagnoses in patients with hypo/hyperthyroidism. We found an absolute cancer risk of 3.0\%-3.9\% in the first year following VTE and a relative cancer risk of 2.0-2.7 compared with the general population. The relative risk of cancer declined after the first year of follow-up, suggesting that development of a VTE can be regarded as a potential early manifestation of an underlying malignancy among patients with hypo/ hyperthyroid disease.

Our study adds to the literature on VTE and cancer by clarifying the association for patients with hypo/hyperthyroidism, in whom thyroid disease itself may cause coagulation disturbances and increase VTE risk, independent of underlying cancer. Similar to previous studies in the general population, we observed the highest increase in cancer risk immediately after VTE diagnosis, followed by a decline. ${ }^{14,16-18}$
One-year SIRs in our study were consistent with the 2- to 4-fold increased cancer risk observed in studies of VTE in the general population. ${ }^{14-18}$

The persistent but modest increase in cancer risk beyond 1 year of follow-up also has been observed for VTE patients in general ${ }^{14,16-18}$ and may be explained by common shared lifestyle risk factors for VTE and cancer, such as smoking, obesity, or hormone replacement therapy, or by premalignant changes that promote thrombosis. ${ }^{17}$ Another possible explanation for our results is the putative oncogenic effects of thyroid hormones. ${ }^{31}$

Awareness of the association between VTE and cancer may lead to heightened diagnostic efforts. This is suggested by the higher SIRs observed in the later calendar-year period. However, a period of increased cancer risk then would have been followed by a compensatory deficit, ${ }^{17}$ which was not observed. This implies that detection bias does not explain our results.

The number of patients needed to examine to detect 1 excess cancer within the first year after VTE was only 30 in the hyperthyroidism subcohort and 52 in the hypothyroidism subcohort. However, the clinical utility of extensive screening for cancer in VTE patients depends on the ability to detect the cancer using these methods, as well as on the prognostic impact of earlier cancer detection. These topics were not investigated in this study. Cancers preceded by VTE have a higher stage at diagnosis and a worse prognosis compared with other cancers. ${ }^{32}$ Previous studies have not provided strong evidence that extensive screening to detect occult cancer after VTE improves patient prognosis. ${ }^{33-36}$ Moreover, extensive screening for cancer may be associated with physical and psychological discomfort. ${ }^{37}$

Still, detection of an underlying cancer may have implications for VTE management, including treatment of the VTE. ${ }^{38}$ We, thus, concur that patients with hypo/hyperthyroidism and VTE undergo diagnostic workup for cancer to the same extent as non-thyroid patients diagnosed with VTE.

The validity of our results depends on several factors. Major study strengths are its nationwide population-based design and completeness of patient follow-up, which reduced the risk of selection bias. As the DNPR covers all hospital contacts in Denmark, the study was not affected by selective inclusion of specific hospitals, health insurance systems, or age groups. As well, the validity of data on VTE, ${ }^{22}$ thyroid disease,$^{39}$ cancer $^{40}$ and comorbidities ${ }^{41}$ is high.

Study limitations include the potential for protopathic bias, that is, cancer diagnostic activities leading to a VTE diagnosis. Moreover, in some cases, the registration of a 
cancer diagnosis might be delayed compared with a concomitant VTE diagnosis. However, in our study, an increased cancer risk was still observed after excluding cancers detected within 30 days post-VTE. Our cancer site-specific results should be interpreted with caution since analysis of the highrisk cancer sites were based on small numbers of cancers, limiting statistical precision. We lacked drug utilization data and could, therefore, not stratify our cancer analyses of thyroid disease patients by use of VTE-associated drugs. Finally, we lacked biochemical data and thus, could not evaluate the association between VTE and cancer stratified by thyroid hormone levels. This may be relevant since some studies have indicated that coagulation disturbances in patients with thyroid disease become more marked with increasing deviation of thyroid hormone levels from the normal range. ${ }^{6,8}$ However, restricting our cohort to patients with a more recent thyroid disease diagnosis did not change the results for the hypothyroidism subcohort. For the hyperthyroidism subcohort, the SIRs increased rather than decreased, as would be expected if the hormone disturbances caused more VTEs.

\section{Conclusion}

In conclusion, VTE is a multicausal disease and our findings suggest that among patients with hypothyroidism or hyperthyroidism, VTE also may be a marker of underlying cancer, consistent with observations in the general population. Thus, our results support that patients with hypo/hyperthyroidism and VTE undergo diagnostic workup for cancer to the same extent as non-thyroid patients diagnosed with VTE.

\section{Acknowledgments}

This work was supported by the Danish Cancer Society (grant number R73-A4284-13-S17) and the Program for Clinical Research Infrastructure established by the Lundbeck Foundation and the Novo Nordisk Foundation.

\section{Author contributions}

All authors are responsible for the study design. KV performed the analyses. DHC wrote the initial draft. All authors participated in discussing and interpreting the results. All authors critically revised the manuscript for intellectual content and approved the final version before submission. All authors contributed toward data analysis, drafting and revising the paper and agree to be accountable for all aspects of the work.

\section{Disclosure}

The Department of Clinical Epidemiology, Aarhus University Hospital, receives funding for other studies from companies in the form of research grants to (and administered by) Aarhus University. None of these studies had any relation to the present study.The salary of Diana Christensen is paid by the International Diabetic Neuropathy Consortium (IDNC) research programme, which is supported by a Novo Nordisk Foundation Challenge Programme grant ([Grant number NNF14OC0011633)]. The authors report no other conflicts of interest in this work.

\section{References}

1. Carle A, Laurberg P, Pedersen IB, et al. Epidemiology of subtypes of hypothyroidism in Denmark. Eur J Endocrinol. 2006;154(1):21-28.

2. Cooper DS. Hyperthyroidism. Lancet. 2003;362(9382):459-468.

3. Erem C, Ersoz HO, Karti SS, et al. Blood coagulation and fibrinolysis in patients with hyperthyroidism. $J$ Endocrinol Invest. 2002;25(4):345-350.

4. Stuijver DJ, van Zaane B, Romualdi E, Brandjes DP, Gerdes VE, Squizzato $\mathrm{A}$. The effect of hyperthyroidism on procoagulant, anticoagulant and fibrinolytic factors: a systematic review and meta-analysis. Thromb Haemost. 2012;108(6):1077-1088.

5. Dekkers OM, Erzsebet Horvath-Puho E, Cannegieter SC, Vandenbroucke J, Sorensen HT, Jorgensen JO. Acute cardiovascular events and all-cause mortality in patients with hyperthyroidism: population-based cohort study. Eur J Endocrinol. 2017;176(1):1-9.

6. Debeij J, Dekkers OM, Asvold BO, et al. Increased levels of free thyroxine and risk of venous thrombosis in a large population-based prospective study. J Thromb Haemost. 2012;10(8):1539-1546.

7. Kootte RS, Stuijver DJ, Dekkers OM, et al. The incidence of venous thromboembolism in patients with overt hyperthyroidism: a retrospective multicentre cohort study. Thromb Haemost. 2012;107(3):417-422.

8. van Zaane B, Squizzato A, Huijgen R, et al. Increasing levels of free thyroxine as a risk factor for a first venous thrombosis: a case-control study. Blood. 2010;115(22):4344-4349.

9. Franchini M, Lippi G, Targher G. Hyperthyroidism and venous thrombosis: a casual or causal association? A systematic literature review. Clin Appl Thromb Hemost. 2011;17(4):387-392.

10. Lin HC, Yang LY, Kang JH. Increased risk of pulmonary embolism among patients with hyperthyroidism: a 5-year follow-up study. J Thromb Haemost. 2010;8(10):2176-2181.

11. Franchini M. Hemostatic changes in thyroid diseases: haemostasis and thrombosis. Hematology. 2006;11(3):203-208.

12. Danescu LG, Badshah A, Danescu SC, et al. Venous thromboembolism in patients hospitalized with thyroid dysfunction. Clin Appl Thromb Hemost. 2009;15(6):676-680.

13. Goldhaber SZ, Bounameaux H. Pulmonary embolism and deep vein thrombosis. Lancet. 2012;379(9828):1835-1846.

14. Sorensen HT, Svaerke C, Farkas DK, et al. Superficial and deep venous thrombosis, pulmonary embolism and subsequent risk of cancer. Eur $J$ Cancer. 2012;48(4):586-593.

15. Iodice S, Gandini S, Lohr M, Lowenfels AB, Maisonneuve P. Venous thromboembolic events and organ-specific occult cancers: a review and meta-analysis. J Thromb Haemost. 2008;6(5):781-788.

16. Murchison JT, Wylie L, Stockton DL. Excess risk of cancer in patients with primary venous thromboembolism: a national, population-based cohort study. Br J Cancer. 2004;91(1):92-95.

17. Baron JA, Gridley G, Weiderpass E, Nyren O, Linet M. Venous thromboembolism and cancer. Lancet. 1998;351(9109):1077-1080.

18. Sorensen HT, Mellemkjaer L, Steffensen FH, Olsen JH, Nielsen GL. The risk of a diagnosis of cancer after primary deep venous thrombosis or pulmonary embolism. $N$ Engl J Med. 1998;338(17):1169-1173.

19. Prandoni P, Lensing AW, Buller HR, et al. Deep-vein thrombosis and the incidence of subsequent symptomatic cancer. $N$ Engl $J$ Med. 1992;327(16):1128-1133. 
20. Schmidt M, Pedersen L, Sorensen HT. The Danish Civil Registration System as a tool in epidemiology. Eur J Epidemiol. 2014;29(8): 541-549.

21. Lynge E, Sandegaard JL, Rebolj M. The Danish National Patient Register. Scand J Public Health. 2011;39(7 Suppl):30-33.

22. Sundboll J, Adelborg K, Munch T, et al. Positive predictive value of cardiovascular diagnoses in the Danish National Patient Registry: a validation study. BMJ Open. 2016;6(11):e012832.

23. Severinsen MT, Kristensen SR, Overvad K, Dethlefsen C, Tjonneland A, Johnsen SP. Venous thromboembolism discharge diagnoses in the Danish National Patient Registry should be used with caution. J Clin Epidemiol. 2010;63(2):223-228.

24. Gjerstorff ML. The Danish Cancer Registry. Scand J Public Health. 2011;39(7 Suppl):42-45.

25. Charlson ME, Pompei P, Ales KL, MacKenzie CR. A new method of classifying prognostic comorbidity in longitudinal studies: development and validation. J Chronic Dis. 1987;40(5):373-383.

26. Ording AG, Sorensen HT. Concepts of comorbidities, multiple morbidities, complications, and their clinical epidemiologic analogs. Clin Epidemiol. 2013;5:199-203.

27. Kim HT. Cumulative incidence in competing risks data and competing risks regression analysis. Clin Cancer Res. 2007;13(2 Pt 1):559-565.

28. Breslow NE, Day NE. Statistical methods in cancer research. Volume II - The design and analysis of cohort studies. IARC Sci Publ. 1987;82:69-72.

29. Kirkwood B, Sterne J. Standardization. In: Goodgame F, editor. Medical Statistics. 2nd ed. Oxford: Blackwell Publishing; 2003:263-271.

30. Altman DG. Confidence intervals for the number needed to treat. $B M J$ 1998;317(7168):1309-1312.

31. Hellevik AI, Asvold BO, Bjoro T, Romundstad PR, Nilsen TI, Vatten LJ. Thyroid function and cancer risk: a prospective population study. Cancer Epidemiol Biomarkers Prev. 2009;18(2):570-574.
32. Sorensen HT, Mellemkjaer L, Olsen JH, Baron JA. Prognosis of cancers associated with venous thromboembolism. $N$ Engl J Med. 2000;343(25):1846-1850.

33. Piccioli A, Lensing AW, Prins MH, et al; SOMIT Investigators Group. Extensive screening for occult malignant disease in idiopathic venous thromboembolism: a prospective randomized clinical trial. J Thromb Haemost. 2004;2(6):884-889.

34. Van Doormaal FF, Terpstra W, Van Der Griend R, et al. Is extensive screening for cancer in idiopathic venous thromboembolism warranted? J Thromb Haemost. 2011;9(1):79-84.

35. Robertson L, Yeoh SE, Stansby G, Agarwal R. Effect of testing for cancer on cancer- and venous thromboembolism (VTE)-related mortality and morbidity in patients with unprovoked VTE. Cochrane Database Syst Rev. 2015;3:CD010837.

36. Carrier M, Lazo-Langner A, Shivakumar S, et al; SOME Investigators Screening for occult cancer in unprovoked venous thromboembolism. N Engl J Med. 2015;373(8):697-704.

37. Prins MH, Lensing AW, Hirsh J. Idiopathic deep venous thrombosis. Is a search for malignant disease justified? Arch Intern Med. 1994;154(12):1310-1312.

38. Shaboodien R, Stansby G, Hunt BJ, Agarwal R. Unprovoked venous thromboembolism: assess for cancer. Lancet Oncol. 2012;13(10):973-974.

39. Vestergaard P, Mosekilde L. Fractures in patients with hyperthyroidism and hypothyroidism: a nationwide follow-up study in 16,249 patients. Thyroid. 2002;12(5):411-419.

40. Storm HH, Michelsen EV, Clemmensen IH, Pihl J. The Danish Cancer Registry-history, content, quality and use. Dan Med Bull. 1997;44(5):535-539.

41. Thygesen SK, Christiansen CF, Christensen S, Lash TL, Sorensen HT. The predictive value of ICD-10 diagnostic coding used to assess Charlson comorbidity index conditions in the population-based Danish National Registry of Patients. BMC Med Res Methodol. 2011;11:83.
Clinical Epidemiology

\section{Publish your work in this journal}

Clinical Epidemiology is an international, peer-reviewed, open access, online journal focusing on disease and drug epidemiology, identification of risk factors and screening procedures to develop optimal preventative initiatives and programs. Specific topics include: diagnosis, prognosis, treatment, screening, prevention, risk factor modification,

Submit your manuscript here: https://www.dovepress.com/clinical-epidemiology-journal

\section{Dovepress}

systematic reviews, risk and safety of medical interventions, epidemiology and biostatistical methods, and evaluation of guidelines, translational medicine, health policies and economic evaluations. The manuscript management system is completely online and includes a very quick and fair peer-review system, which is all easy to use. 